\title{
Patient Satisfaction and Clinical Efficacy of Flash Glucose Monitoring in Patients with Type 1 Diabetes: A Prospective, Single-Center, Single-Arm Study
}

\author{
Keisuke Ueno - Daisuke Chujo (D) - Nobuyuki Takahashi · \\ Mitsuru Ohsugi · Kohjiro Ueki · Hiroshi Kajio
}

Received: May 2, 2020 / Published online: June 23, 2020

(C) The Author(s) 2020
Digital Features To view digital features for this article go to https://doi.org/10.6084/m9.figshare.12417821.

Electronic Supplementary Material The online version of this article (https://doi.org/10.1007/s13300020-00859-1) contains supplementary material, which is available to authorized users.

K. Ueno - D. Chujo $(\bowtie) \cdot$ N. Takahashi · M. Ohsugi · K. Ueki · H. Kajio

Department of Diabetes, Endocrinology and

Metabolism, National Center for Global Health and

Medicine, Tokyo, Japan

e-mail: dchujo@med.u-toyama.ac.jp

K. Ueno

Department of Diabetes and Endocrinology, Japan Health Care Organization Tokyo Shinjuku Medical Center, Tokyo, Japan

D. Chujo

Center for Clinical Research, Toyama University

Hospital, Toyama, Japan
Methods: Patient satisfaction with FGM was assessed using the Diabetes Treatment Satisfaction Questionnaire (DTSQ) and Diabetes Therapy-Related Quality of Life (DTR-QOL) questionnaire before (baseline) and 4 and 12 weeks after initiating FGM use in 20 Japanese patients with T1D. Clinical parameters related to glucose metabolism, such as glycated hemoglobin (HbA1c) levels, were assessed, and glucose fluctuations were evaluated using the FGM data. Values at 4 and 12 weeks after initiating FGM were compared with baseline data using the Wilcoxon signed-rank test. The mean absolute relative difference (MARD) between glucose values detected using the FGM device and by SMBG was also calculated.

Results: The DTSQ scores significantly improved 12 weeks after initiating FGM $(P<0.001)$. The DTR-QOL scores related to "burden in social activities" and "treatment satisfaction" also significantly improved 12 weeks after initiating FGM $(P=0.024$ and 0.007 , respectively). The HbA1c values and the percentage of time within the target glucose range $(3.9-7.8 \mathrm{mmol} / \mathrm{L})$ at the 12 -week time point also improved, from $58 \pm 5$ (baseline) to $54 \pm 4 \mathrm{mmol} / \mathrm{mol}(P=0.002)$ and from $36 \%$ (interquartile range [IQR] 25-44.5\%) (baseline) to $43 \%$ (IQR 39-50\%) $(P=0.016)$, respectively. The MARD between glucose values detected using FGM and those detected using SMBG was $12.2 \%$ throughout the study period. 
Conclusions: Flash glucose monitoring contributed to improved patient satisfaction and the adjustment of blood glucose levels in patients with T1D.

Trial Registration: University Hospital Medical Information Network Clinical Trials Registry (UMIN-CTR): UMIN000029673

Keywords: Flash glucose monitoring; Quality of life; Satisfaction; Type 1 diabetes

\section{Key Summary Points}

Why carry out this study?

Flash glucose monitoring (FGM), a lessinvasive glucose monitoring method, has launched in Japan.

This study aimed to evaluate patient satisfaction, by using the established questionnaires, and the clinical efficacy of FGM in the patients with type 1 diabetes (T1D).

\section{What was learned from the study?}

The scores of the questionnaires related to quality of life (QOL) in T1D patients were significantly improved 12 weeks after using FGM.

The values of glycated hemoglobin (HbA1c) and the percentages of time within the target glucose range (3.9-7.8 $\mathrm{mmol} / \mathrm{L}$ ) also improved at 12 weeks after using FGM.

Our study suggests that FGM contributed to improving patient satisfaction and the adjustment of blood glucose levels in T1D patients.

\section{INTRODUCTION}

Patients with diabetes usually experience incessant stress related to dietary restrictions, medical expenses, complications, among others, which often leads to a negative impact on their quality of life (QOL). Studies have shown that patients with diabetes are more susceptible to depression than persons without diabetes [1-4]. In particular, patients with type 1 diabetes (T1D) experience considerable psychological burden due to unexpected fluctuations in blood glucose levels, multiple daily insulin injections, and the need for frequent self-monitoring of blood glucose (SMBG) [5]. An additional challenge is that patients with T1D often encounter difficulty in achieving optimal glycemic control due to the lack of endogenous insulin secretion and imprecise insulin dosing. Previous studies have shown that only $20 \%$ of these patients achieve a glycated hemoglobin (HbA1c) level of $<52 \mathrm{mmol} / \mathrm{mol} \quad(<7.0 \%)$ $[6,7]$. Although frequent SMBG usually results in improved glycemic control $[8,9]$, in some patients the frequency of SMBG is low due to their fear of self-testing or their low pain threshold. These factors may affect patients' decisions and behavior regarding the monitoring of their glucose levels [10]. Hygiene and social obstacles in their schools or workplaces may also deter patients from SMBG.

Following the introduction of continuous glucose monitoring (CGM) systems in clinical practice, it became possible to visualize glucose trends by placing a sensor under the skin. This device is beneficial in terms of identifying hypoglycemia, especially during sleep and between meals, but finger-prick blood sampling is still necessary for calibration purposes. Flash glucose monitoring (FGM) was launched in Japan in September 2017. FGM is a less-invasive glucose monitoring method than SMBG because patients do not need to prick their fingers. The patients can access real-time glucose levels by holding the reader over the sensor placed under their skin, without the need for calibration. Bolinder et al. reported that glycemic control in patients with T1D improved following initiation of FGM [11].

This less-invasive FGM device can be very useful, especially for patients with T1D who require frequent measurements of glucose levels. Therefore, we hypothesized that FGM could improve the QOL of T1D patients. Since few previous studies have evaluated patient satisfaction as the primary outcome, we 
performed a prospective study to evaluate patient satisfaction and changes in glycemic control following the introduction of FGM in Japanese patients with T1D.

\section{METHODS}

\section{Study Design and Ethics}

This was a prospective, single-center, single-arm study conducted at the National Center for Global Health and Medicine between January 2017 and January 2018. All procedures performed in studies involving human participants were in accordance with the ethical standards of the institutional and/or national research committee and with the 1964 Helsinki Declaration and its later amendments or comparable ethical standards. The study protocol was approved by the ethical committee of the National Center for Global Health and Medicine (ID: NCGM-G002141-00). Written informed consent was obtained from all the participants.

\section{Participants and Study Assessment}

Outpatients with T1D and aged 18-75 years were recruited to the study. All patients had to be able to clearly understand the consent form and be capable of providing written consent. Patients with skin disorders were excluded. For FGM, the Free style Libre ${ }^{\circledR}$ (Abbott Medical Japan, LLC, Tokyo, Japan) device was used. The FGM sensors remained attached to the study participants throughout the study period. Patient satisfaction with FGM was assessed using questionnaires before the initiation of FGM (baseline) and at 4 and 12 weeks after initiation of FGM. Also, clinical parameters related to glucose metabolism, such as HbA1c, were obtained from blood analyses, and fluctuations in glucose levels were evaluated using the FGM data. The target range for the blood glucose level was 3.9 to $7.8 \mathrm{mmol} / \mathrm{L}$, and the whole measurement period was divided into three target ranges: $<3.9,3.9-7.8$, and $>7.8 \mathrm{mmol} / \mathrm{L}$. The correlation between glucose values obtained using the FGM device and those obtained with SMBG was also investigated for 5 days. Throughout the study period, all patients continued on their insulin therapy, and the dosage of insulin was adjusted to target glucose levels between 5.6 and $7.8 \mathrm{mmol} / \mathrm{L}$ before each meal.

\section{Assessment of Patient Satisfaction with FGM Using Questionnaires}

Patient satisfaction with FGM was assessed using the Japanese language versions of the Diabetes Treatment Satisfaction Questionnaire (DTSQ) and the Diabetes Therapy-Related Quality of Life (DTR-QOL) questionnaire [12-14]. The DTSQ is composed of eight questions, six of which assess treatment satisfaction. Each question is scored by a patient on a 7-point scale (range 0-6). The six questions on treatment satisfaction ask about "satisfaction with current treatment," "flexibility," "convenience," "understanding of diabetes," "recommend treatment to others," and "willingness to continue," respectively. Treatment satisfaction is evaluated as the summary of scores of the six questions (best total score 36) [12, 13]. The DTRQOL questionnaire assesses the influence of diabetes treatment on the patient's QOL. This questionnaire has 29 items, which are divided into four groups: "burden on social activities and daily activities" (13 items), "anxiety and dissatisfaction with treatment" (8 items), "hypoglycemia" (4 items), and "satisfaction with treatment" (4 items) [14]. In both questionnaires, higher scores indicate higher satisfaction with the treatment.

\section{Statistical Analysis}

Data are presented as the mean value \pm standard deviation (SD) or the median value and interquartile range (IQR). Scores from the DTSQ and DTR-QOL questionnaire, values for clinical parameters related to glucose metabolism, and fluctuations in glucose at 12 weeks after FGM initiation were compared with those at baseline. The Wilcoxon signed ranked test was used to assess the difference between the two groups. For the analysis, $P<0.05$ was considered to be 
statistically significant. When evaluating the correlation between glucose values obtained using the FGM device and those obtained by SMBG, Spearman's correlation and the mean absolute relative difference (MARD) were calculated. Statistical analysis was conducted with STATA software, version 14.0 (StataCorp, College Station, TX, USA).

\section{RESULTS}

\section{Baseline Characteristics}

A total of 21 patients with T1D participated in this study. One patient was excluded due to skin eruptions, and the remaining 20 patients were included in the final analysis. As seen in Electronic Supplementary Material (ESM) Table S1, the mean $( \pm S D)$ age of the participants was $54.6 \pm 14.4$ years, and mean diabetes duration was $9.1 \pm 11.8$ years. Of the 20 patients in the final analysis, 15 received multiple daily insulin injection therapy, and five received insulin pump therapy. Prior to using the FGM device, all participants performed SMBG 4 times a day, once before each meal and before going to bed.

\section{DTSQ and DTR-QOL Scores Improved After the Introduction of FGM}

Following the introduction of FGM, the DTSQ scores were found to have significantly improved from $21.9 \pm 8.1$ at baseline to $31.5 \pm 3.6$ at 4 weeks $(P<0.001)$ and $32.4 \pm 3.8$ at 12 weeks $(P<0.001)$ (Fig. 1 a). The DTR-QOL scores in all four groups of question items had also significantly improved at 4 weeks after the introduction of FGM, with the scores for "burden in social activities," "anxiety and dissatisfaction with treatment," "hypoglycemia," and "satisfaction with treatment" increasing from $52.8 \pm 26.3$ (before FGM) to $71.0 \pm 23.0(P=0.009)$, from $47.2 \pm 24.4$ to $57.3 \pm 23.2(P=0.047)$, from $34.8 \pm 25.2$ to $55.5 \pm 29.3(P=0.046)$, and from $48.3 \pm 25.3$ to $65.6 \pm 22.9(P=0.006)$, respectively. At 12 weeks after initiating FGM, the scores related to "burden in social activities" and "treatment
Fig. 1 Patient scores for the Diabetes Treatment Satisfaction Questionnaire $(D T S Q)(\mathbf{a})$ and Diabetes TherapyRelated Quality of Life (DTR-QOL) questionnaire (b) before and 4 and 12 weeks after the initiation of Flash glucose monitoring. Higher scores indicate higher satisfaction with the treatment. FGM Flash glucose monitoring. *Significantly different at $P<0.05$ compared with the scores before the introduction of FGM

satisfaction with treatment" were significantly higher compared to the scores before the introduction of FGM, increasing from $52.8 \pm 26.3$ to $67.5 \pm 24.7(P=0.024)$ and from $48.3 \pm 25.3$ to $69.5 \pm 23.7(P=0.007)$, respectively (Fig. 1b).

\section{HbA1c Levels and Percentage of Time Spent in Target Glucose Range Improved After the Introduction of FGM}

The HbA1c levels significantly decreased from $58 \pm 5 \mathrm{mmol} / \mathrm{mol}(7.46 \pm 0.66 \%)$ at baseline to $56 \pm 5 \mathrm{mmol} / \mathrm{mol} \quad(7.32 \pm 0.66 \%) \quad(P=0.041)$ and $\quad 54 \pm 4 \mathrm{mmol} / \mathrm{mol} \quad(7.16 \pm 0.58 \%)$ $(P=0.002)$ at 4 and 12 weeks after the introduction of FGM, respectively (Table 1). Moreover, at 12 weeks, the standard deviation of glucose levels obtained from the FGM data improved significantly from $3.6 \pm 0.9$ to $3.3 \pm 1.0 \mathrm{mmol} / \mathrm{L}(P=0.035)$, as did the percentage of time spent within the target glucose range (3.9-7.8 $\mathrm{mmol} / \mathrm{L})$ at 12 weeks after initiating FGM. The percentage of time in glucose levels below and above the target range were not significantly changed after the introduction of FGM.

\section{Correlation Between Glucose Values Obtained Using FGM and Those Obtained by SMBG}

Glucose values obtained using the FGM device were significantly correlated with those obtained by SMBG $\left(R^{2}=0.881, P<0.0001\right)$. The MARD between glucose values obtained by FGM and SMBG was $12.2 \%$ throughout the study period (ESM Fig. S1). 
a

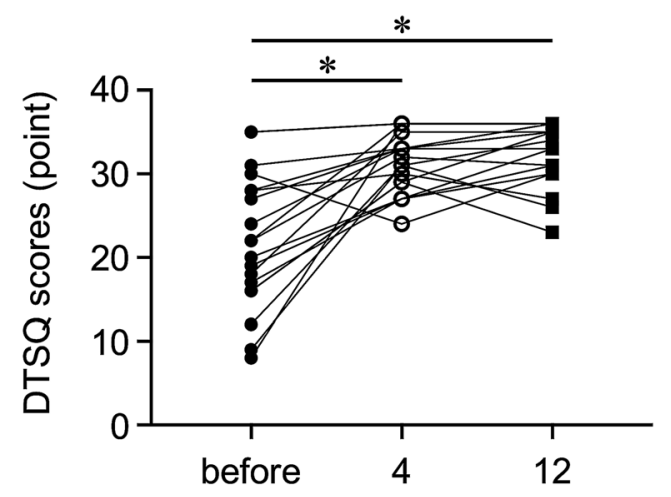

Weeks after the introduction of FGM

b
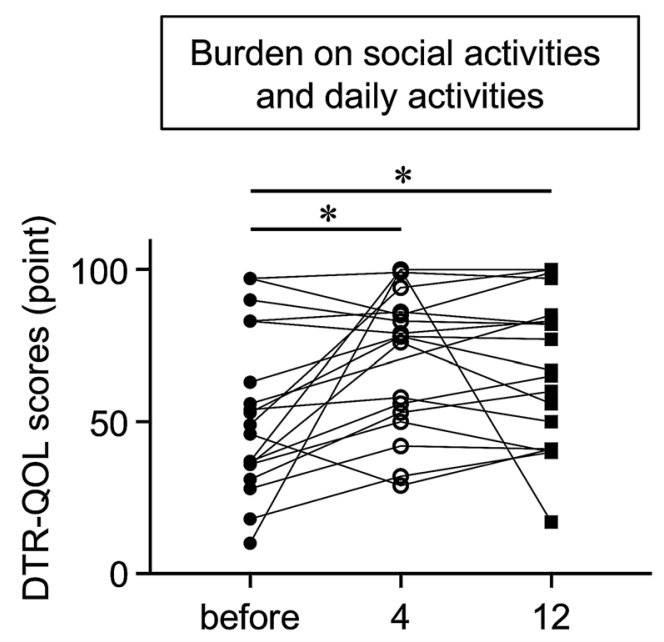

Weeks after the introduction of FGM

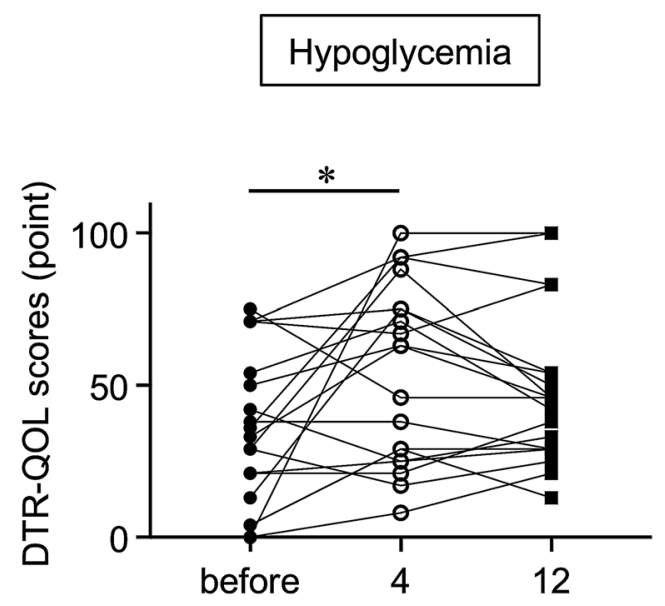

Weeks after the introduction of FGM
Anxiety and dissatisfaction with treatment

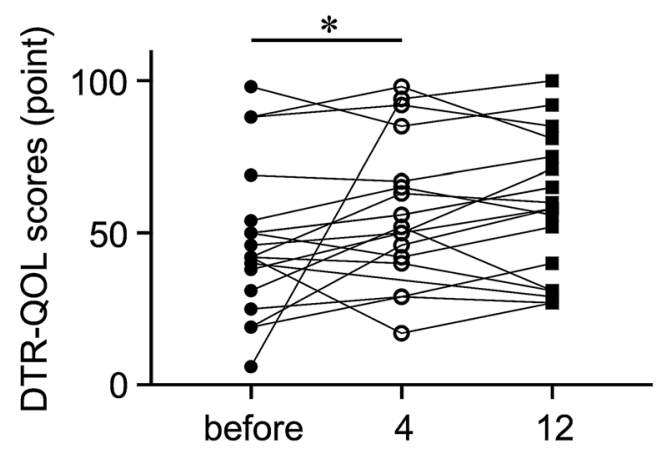

Weeks after the introduction of FGM

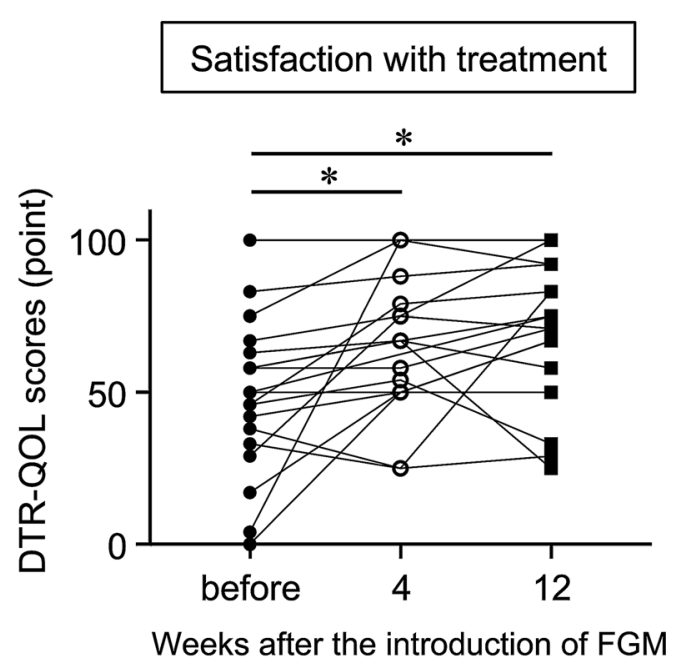


Table 1 Glycated hemoglobin levels and results of flash glucose monitoring (FGM) analysis before and 4 and 12 weeks after the introduction of FGM

\begin{tabular}{|c|c|c|c|}
\hline Parameters & Before FGM & 4 weeks after initiating FGM & 12 weeks after initiating FGM \\
\hline $\mathrm{HbAlc}(\mathrm{mmol} / \mathrm{mol})$ & $58 \pm 5$ & $56 \pm 5^{*}$ & $54 \pm 4^{*}$ \\
\hline Average glucose $(\mathrm{mmol} / \mathrm{L})$ & $9.3 \pm 1.9$ & $9.0 \pm 1.5$ & $8.7 \pm 1.2$ \\
\hline $\mathrm{SD}$ of glucose $(\mathrm{mmol} / \mathrm{L})$ & $3.6 \pm 0.9$ & $3.4 \pm 0.9$ & $3.3 \pm 1.0^{*}$ \\
\hline \multicolumn{4}{|c|}{ Percentage of time spent within the range of glucose levels } \\
\hline$<3.9 \mathrm{mmol} / \mathrm{L}$ & $4(1-5.5)$ & $4(1-6)$ & $2(0-10)$ \\
\hline $3.9-7.8 \mathrm{mmol} / \mathrm{L}$ & $36(25-44.5)$ & $41(33-52.5)$ & $44(39-50)^{*}$ \\
\hline$>7.8 \mathrm{mmol} / \mathrm{L}$ & $60(47-69.5)$ & $55(44.5-65.5)$ & $54(42.5-61.5)$ \\
\hline
\end{tabular}

Data are expressed as the mean \pm standard deviation (SD) for glycated hemoglobin (HbAlc), average glucose, and SD of glucose, and as median with the interquartile range in parenthesis for the percentage of time spent within the range of glucose levels

${ }^{*}$ Significant difference at $P<0.05$ compared with the values before the introduction of FGM

\section{Correlation Between the Frequency of Glucose Scanning with the FGM Device and Changes in DTSQ Scores and HbA1c Levels}

The frequency of glucose scanning with FGM was not significantly correlated with changes in either DTSQ scores (ESM Fig. S2a) or HbA1c levels (ESM Fig. S2b) during the study period ( $R^{2}=0.055$ and $R^{2}<0.001$, respectively).

\section{DISCUSSION}

The results of our study demonstrated that FGM contributed to improved patient satisfaction, as evaluated by two questionnaires related to QOL and glycemic control during the 12-week study period in patients with T1D. The accuracy of FGM was also reconfirmed in our study.

The DTSQ scores significantly improved from $21.9 \pm 8.1$ at baseline to $32.4 \pm 3.8$ at 12 weeks after the introduction of FGM. The changes in the DTSQ scores in our study are relatively larger than those reported in other studies. For example, Akiko et al. reported that the scores improved from 19.4 to 23.9 when the carbohydrate counting system was introduced in patients with T1D [15]. The DTR-QOL scores in our study were especially related to "burden on social and daily activities" and "satisfaction with treatment," and the scores for these two groups were also significantly higher following the initiation of FGM. These high scores may be related to the ease and low invasiveness of FGM. The DTR-QOL scores related to "anxieties and dissatisfactions" and "hypoglycemia" also improved 4 weeks after the introduction of FGM; these scores were low in one-third of the participants. While the patients could easily access real-time glucose levels with FGM any time they wanted, some of them felt more anxious about hypoglycemia and hyperglycemia when they saw several values of glucose levels. These results suggest that the personality and character of a patient needs be taken into consideration during decision-making on whether to initiate FGM.

Interestingly, the $\mathrm{HbA1c}$ levels of the patients in our study significantly improved during the study period, in contrast to results reported by Bolinder et al. which showed that FGM reduced the time spent in and incidence of hypoglycemia, but did not decrease the HbA1c levels [11]. The main reason for this difference may be that the mean initial HbA1c levels of the patients in our study were higher than those of patients included in the earlier study (58 vs. $48 \mathrm{mmol} / \mathrm{mol}$ [7.46 vs. $6.70 \%]$ ). This possibility is supported by a study by Evans et al. who found that changes in HbA1c levels are affected by the initial values [16]. Conversely, a number of 
other recent studies have shown that the introduction of FGM contributed to the reduction of HbA1c levels, similar to what we demonstrated in our study $[16,17]$. Furthermore, in our study, the SD of glucose levels and the percentage of time spent in target glucose range had improved without any increase in the percentage of time in hypoglycemia. These results were possible because the patients could perform certain interventions, such as modifying glucose intake or an additional bolus insulin injection, by monitoring their glucose trend with the FGM device, thereby avoiding both hypoglycemia and hyperglycemia. Accordingly, FGM can be a good option for managing T1D because achieving better HbA1c levels, avoiding hypoglycemia, and regulating glucose fluctuations are regarded as important therapeutic goals [18].

Our study had a number of limitations. First, it was an exploratory study with a single-arm, a relatively low number of participants, and a short observation period. Large changes in the QOL scores in a few patients might therefore affect the results in a study with small number of participants. Second, since the participants were older than 18 years and not pregnant, it is unclear whether FGM can be a satisfactory option for patients in the younger age group and pregnant women, who might behave differently from the participants of our study. Third, although the target blood glucose level was $3.9-7.8 \mathrm{mmol} / \mathrm{L}$ before each meal, we did not use any strict algorithm for insulin adjustment. Therefore, randomized studies with a higher number of patients, longer duration, and strict algorithms for insulin adjustment should be considered in the future.

\section{CONCLUSIONS}

The results of our study suggest that FGM contributes to improving patient satisfaction and enables a better adjustment of glucose levels in patients with T1D.

\section{ACKNOWLEDGEMENTS}

The authors thank all of the investigators and patients who took part in this study.
Funding. This study and the journal's Rapid Service Fee were supported by a grant from the National Center for Global Health and Medicine (Grant number: 28S1204). All authors had full access to all of the data in this study and take complete responsibility for the integrity of the data and accuracy of the data analysis.

Editorial Assistance. The authors thank Prafulla Rai and Carol Metcalf of Editage (Tokyo, Japan; www.editage.jp) for English language editing. This assistance was funded by National Center for Global Health and Medicine (Grant number: 28S1204).

Authorship. All named authors meet the International Committee of Medical Journal Editors (ICMJE) criteria for authorship for this article, take responsibility for the integrity of the work as a whole, and have given their approval for this version to be published.

Prior Presentation. A part of this study was presented at the 12th International Conference on Advanced Technologies and Treatments for Diabetes (ATTD 2019) in Berlin, Germany on 20-23 February 2019.

Disclosures. Keisuke Ueno, Nobuyuki Takahashi, Mitsuru Ohsugi, Kohjiro Ueki, Hiroshi Kajio, and Daisuke Chujo have nothing to disclose.

Compliance with Ethics Guidelines. All procedures performed in studies involving human participants were in accordance with the ethical standards of the institutional and/or national research committee and with the 1964 Helsinki Declaration and its later amendments or comparable ethical standards. The study protocol was approved by the ethical committee of the National Center for Global Health and Medicine (ID: NCGM-G-002141-00). Written informed consent was obtained from all the participants.

Data Availability. The data sets generated and/or analyzed during the current study are available from the corresponding author on reasonable request. 
Open Access. This article is licensed under a Creative Commons Attribution-NonCommercial 4.0 International License, which permits any non-commercial use, sharing, adaptation, distribution and reproduction in any medium or format, as long as you give appropriate credit to the original author(s) and the source, provide a link to the Creative Commons licence, and indicate if changes were made. The images or other third party material in this article are included in the article's Creative Commons licence, unless indicated otherwise in a credit line to the material. If material is not included in the article's Creative Commons licence and your intended use is not permitted by statutory regulation or exceeds the permitted use, you will need to obtain permission directly from the copyright holder. To view a copy of this licence, visit http://creativecommons.org/licenses/by$\mathrm{nc} / 4.0 /$.

\section{REFERENCES}

1. Peyrot M, Rubin RR, Lauritzen T, Snoek FJ, Matthews DR, Skovlund SE. Psychosocial problems and barriers to improved diabetes management: results of the Cross-National Diabetes Attitudes, Wishes and Needs (DAWN) Study. Diabet Med. 2005;22:1379-85.

2. Pouwer F, Hermanns N. Insulin therapy and quality of life A review. Diabetes Metab Res Rev. 2009;25: S4-10.

3. Vancampfort D, Correll CU, Gallinget B, et al. Diabetes mellitus in people with schizophrenia, bipolar disorder and major depressive disorder: a systematic review and large scale meta-analysis. World Psychiatry. 2016;15:166-74.

4. De Groot M, Golden SH, Wagner J. Psychological conditions in adults with diabetes. Am Psychol. 2016;71:552-62.

5. Trief PM, Xing D, Foster NC, et al. T1D exchange clinic network. Depression in adults in the T1D exchange clinic registry. Diabetes Care. 2014;37:1563-72.

6. Foster NC, Beck RW, Miller KM, et al. State of Type 1 diabetes management and outcomes from the T1D exchange 2016-2018. Diabetes Techhnol Ther. 2019;21:66-72.

7. Pettus JH, Zhou FL, Shepherd L, et al. Incidences of severe hypoglycemia and diabetic ketoacidosis and prevalence of microvascular complications stratified by age and glycemic control in U.S. adult patients with type 1 diabetes: a real-world study. Diabetes Care. 2019;42:2220-7.

8. Miller KM, Beck RW, Bergenstal RM, et al. Evidence of a strong association between frequency of selfmonitoring of blood glucose and hemoglobin A1c levels in T1D Exchange clinic registry participants. Diabetes Care. 2013;36:2009-144.

9. Nyomba BL, Berard L, Murphy LJ. Facilitating access to glucometer regents increases blood glucose monitoring frequency and improves glycaemic control: a prospective study in insulin-treated diabetes patients. Diabet Med. 2004;21:129-35.

10. Reach G. Decisions in the psychology of glucose monitoring. J Diabetes Sci Technol. 2019;13:1169-74.

11. Bolinder J, Antuna R, Geelhoed-Duijvestijn P, Kröger J, Weitgasser R. Novel glucose-sensing technology and hypoglycaemia in type 1 diabetes: multicenter, non-masked, randomized controlled trial. Lancet. 2016;388:2254-63.

12. Saisho Y. Use of diabetes treatment satisfaction questionnaire in diabetes care: importance of patient-reported outcomes. Int J Environ Res Public Health. 2018;15:E947.

13. Ishii $\mathrm{H}$, Bradley $\mathrm{C}$, Riazi $\mathrm{A}$, et al. The Japanese version of the diabetes treatment satisfaction questionnaire (DTSQ): translation and clinical evaluation. J Clin Exp Med. 2000;192:809-14.

14. Ishii H. Development and psychometric validation of the diabetes therapy-related QOL (DTR-QOL) questionnaire. J Med Econ. 2012;15:556-63.

15. Akiko N, Manami N, Saeko T, et al. The relationship between the acquisition of carbohydrate counting and improvement in QOL in the patients with intensive insulin therapy. Nihon Senshin Tounyobyo Chiryo Kenkyuukai Zasshi. 2014;9:19-22.

16. Evans M, Welsh Z, Ells S, Seibold A. The impact of flash glucose monitoring on glycaemic control as measured by HbA1c: a meta-analysis of clinical trials and real-world observational studies. Diabetes Ther. 2020;11:83-95.

17. Nana M, Moore SL, Ang E, Lee ZX, Bondugulapati LNR. Flash glucose monitoring: impact on markers of glycaemic control and patient-reported outcomes in individuals with type 1 diabetes mellitus in the real-world setting. Diabetes Res Clin Pract. 2019;157:107893.

18. Gerry R. Glycaemic control, glucose variability and the triangle of diabetes care. Br J Diabetes. 2016;16: S3-S6. 